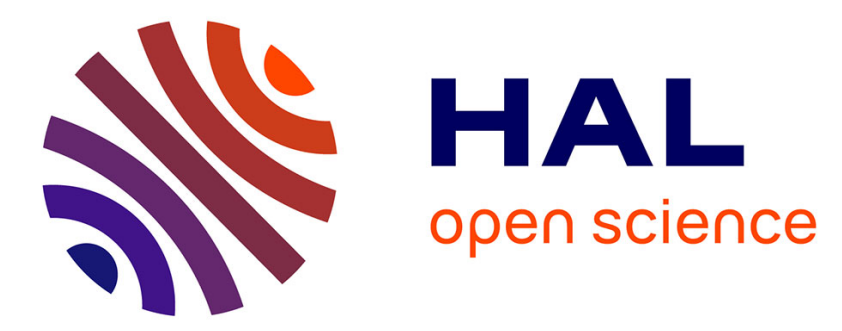

\title{
Fault tolerant control for induction motors using sliding mode observers
}

\author{
Nadia Djeghali, Malek Ghanes, Said Djennoune, Jean-Pierre Barbot
}

\section{To cite this version:}

Nadia Djeghali, Malek Ghanes, Said Djennoune, Jean-Pierre Barbot. Fault tolerant control for induction motors using sliding mode observers. VSS, Jun 2011, Mexico, Mexico. hal-00772797

\section{HAL Id: hal-00772797 https://hal.inria.fr/hal-00772797}

Submitted on 11 Jan 2013

HAL is a multi-disciplinary open access archive for the deposit and dissemination of scientific research documents, whether they are published or not. The documents may come from teaching and research institutions in France or abroad, or from public or private research centers.
L'archive ouverte pluridisciplinaire HAL, est destinée au dépôt et à la diffusion de documents scientifiques de niveau recherche, publiés ou non, émanant des établissements d'enseignement et de recherche français ou étrangers, des laboratoires publics ou privés. 


\title{
Fault Tolerant Control for Induction Motors using Sliding Mode Observers
}

\author{
N. DJEGHALI, M. GHANES, M. TADJINE, S. DJENNOUNE and J. P. BARBOT
}

\begin{abstract}
In this paper a fault tolerant control design based on a sliding mode observer for induction motors is proposed. First, a direct field oriented controller based on backstepping technique is designed in order to steer the flux and speed variables to their desired references and to compensate the load disturbance. Second, a sliding mode observer is designed in order to detect and reconstruct the faults and also to estimate the flux. Then, additional control laws based on the estimates of the faults are designed in order to compensate the faults. Numerical simulations show the effectiveness of the proposed control scheme.
\end{abstract}

\section{INTRODUCTION}

Research on fault tolerant control systems has received a great attention due to significantly increasing demand for reliability, maintainability and survivability of physical plants ([1]-[3]). Fault tolerant control systems have abilities to detect the presence of a fault and eventually to isolate it (fault detection and isolation) and also to reconfigure the control law in order to compensate the effect of the fault and maintain the stability and the control performance (fault tolerance).

Induction Motors (IM) are subjected to rotor and stator faults, such as stator short circuits, broken bars or rings, eccentricity,...etc. As in ([4],[5]), this paper is concerned with the rotor asymmetries caused by broken bars or dynamic eccentricity and stator asymmetries caused by static eccentricity. The presence of rotor and stator asymmetries induces harmonic components in the stator currents with frequencies which are directly related to the kind of the fault (stator or rotor fault) and amplitude and phase which depend on the severity of the fault ([4],[5]).

There are many literatures concerning fault tolerant control of induction motors ([4]-[8]). This paper focuses on the implicit fault tolerant controller proposed in ([4],[5]), where the effects generated by the occurrence of the fault are assumed to be modeled as an exogenous signal given by an autonomous "neutrally stable" system (exosystem). In $([4],[5])$ the design of the fault tolerant controller is based on an internal model which requires to know or to estimate the vector of the frequencies characterizing the faults. This design method becomes very difficult in case of a large vector of frequencies.

N. DJEGHALI and S. DJENNOUNE are with Laboratoire de Conduite et Conception des Systèmes de Production, Université Mouloud MAMMERI de Tizi-Ouzou, B.P.17, 15000, Algérie

M.GHANES and J.P.BARBOT are with ECS, ENSEA, 6 avenue du Ponceau, 95014 Cergy, France

M.TADJINE is with Laboratoire de Commandes des Processus, Ecole Nattionale Polytechnique, Alger, 16000, Algérie
In this work the design of the fault tolerant controller for induction motors is based on the use of sliding mode observers for fault detection and reconstruction ([9]-[11]). The sliding mode observers are used in both linear and nonlinear systems with uncertainties. They permit to reconstruct explicitly the faults by analyzing the dynamic of the estimation error when the sliding mode occurs. In our method, the design of the fault tolerant controller does not require to know or to estimate the vector of frequencies. Moreover, the approach proposed here does not require to know the model of the faults effects and it can also compensate the faults caused by the parameters variations and additive faults in actuator. Whereas, the authors in $([4],[5])$ are limited to stator and rotor asymmetries whose model is known. In this paper, it is shown that a Direct Field Oriented Controller based on the backstepping strategy permits to steer the flux and speed variables to their desired references and to compensate the load torque effect. A sliding mode observer is used to reconstruct the faults and to estimate the flux, then additional control laws based on the resulting faults estimates are designed in order to compensate the faults.

This paper is organized as follows: In Section 2, the induction motor oriented model is presented. Section 3 is devoted to the design of the backstepping controller in un-faulty mode, which is able to steer the flux and speed variables to their desired references and to compensate the load disturbance. Section 4 describes the induction motor model in presence of rotor and stator faults. Section 5 presents the faults reconstruction by using a sliding mode observer. Section 6 presents the design of the fault tolerant controller, while Section 7 gives the simulation results. In Section 8, some conclusion remarks on the proposed fault tolerant control are given.

\section{INDUCTION MOTOR ORIENTED MODEL}

In field oriented control, the flux vector is forced to align with the d-axis $\left(\phi_{q r}=\frac{d \phi_{q r}}{d t}=0\right)$. The resulting induction motor model in the $(d-q)$ reference frame is described by the following state equations [12]:

$$
\begin{aligned}
\frac{d i_{d s}}{d t} & =-a i_{d s}+\omega_{s} i_{q s}+\frac{L_{m}}{\sigma L_{s} L_{r} \tau_{r}} \phi_{d r}+\frac{V_{d s}}{\sigma L_{s}} \\
\frac{d i_{q s}}{d t} & =-a i_{q s}-\omega_{s} i_{d s}-\frac{L_{m}}{\sigma L_{s} L_{r}} P \Omega \phi_{d r}+\frac{V_{q s}}{\sigma L_{s}} \\
\frac{d \phi_{d r}}{d t} & =\frac{L_{m}}{\tau_{r}} i_{d s}-\frac{1}{\tau_{r}} \phi_{d r} \\
\frac{d \Omega}{d t} & =\frac{P L_{m}}{L_{r} J} i_{q s} \phi_{d r}-\frac{f}{J} \Omega-\frac{T}{J}
\end{aligned}
$$

with:

$$
\omega_{s}=P \Omega+\frac{L_{m}}{\tau_{r} \phi_{d r}} i_{q s}
$$




$$
a=\left(\frac{R_{s}}{\sigma L_{s}}+\frac{1-\sigma}{\sigma \tau_{r}}\right)
$$

Where $\sigma$ is the coefficient of dispersion, given by:

$$
\sigma=1-\frac{L_{m}^{2}}{L_{s} L_{r}}
$$

$L_{s}, L_{r}, L_{m}$ are stator, rotor and mutual inductance, respectively. $R_{s}, R_{r}$ are respectively stator and rotor resistance. $\omega_{s}$ is the stator pulsation. $\tau_{r}$ is the rotor time constant $\left(\frac{L_{r}}{R_{r}}\right)$. $P$ is the number of pole pairs. $V_{d s}, V_{q s}$ are stator voltage components. $\phi_{d r}, \phi_{q r}$ are the rotor flux components. $\Omega$ is the mechanical speed. $T$ is the load torque. $i_{d s}, i_{q s}$ are stator current components. $J$ is the moment of inertie of the motor. $f$ is the friction coefficient.

\section{BACKSTEPPING CONTROL DESIGN}

This part deals with the speed and flux control by means of backstepping control. This nonlinear control technique can be applied efficiently to linearise a nonlinear system with the existence of uncertainties, it is usually incorporated with the nonlinear damping to enhance robustness ([13],[14]).

In this work in order to compensate the load disturbance the backstepping technique is used. The idea of backstepping design is to select recursively some appropriate functions of state variables as virtual control inputs for lower dimension subsystems of the overall system. At each step of the backstepping a new virtual control input is designed. When the procedure terminates, the actual control input results which achieves the original design objective by virtue of a final Lyapunov function, which is formed by summing up the Lyapunov functions associated with each individual design step.

\section{A. Step1:Flux control}

The objective is to steer the flux $\phi_{d r}$ to a desired reference $\phi_{d r}^{*}$, let $e_{\phi}=\phi_{d r}-\phi_{d r}^{*}$ be the flux tracking error. The dynamic of $e_{\phi}$ is:

$$
\dot{e}_{\phi}=\frac{L_{m}}{\tau_{r}} i_{d s}-\frac{1}{\tau_{r}} \phi_{d r}-\dot{\phi}_{d r}^{*}
$$

A Lyapunov function is defined as:

$$
V_{\phi}=\frac{1}{2} e_{\phi}^{2}
$$

By deriving (4) we obtain:

$$
\dot{V}_{\phi}=e_{\phi} \dot{e}_{\phi}=e_{\phi}\left(\frac{L_{m}}{\tau_{r}} i_{d s}-\frac{1}{\tau_{r}} \phi_{d r}-\dot{\phi}_{d r}^{*}\right)
$$

To make $\dot{V}_{\phi}$ negative definite, $i_{d s}$ is chosen as virtual element of control for stabilizing the flux, its desired value $i_{d s}^{*}$ is defined as:

$$
i_{d s}^{*}=-\frac{\tau_{r}}{L_{m}} k_{\phi} e_{\phi}+\frac{\phi_{d r}}{L_{m}}+\frac{\tau_{r}}{L_{m}} \dot{\phi}_{d r}^{*}
$$

where $k_{\phi}>0$ is a design parameter.

By setting $i_{d s}=i_{d s}^{*}$ in (5) we get :

$$
\dot{V}_{\phi}=-k_{\phi} e_{\phi}^{2}<0
$$

This implies that $e_{\phi} \rightarrow 0$ or $\phi_{d r} \rightarrow \phi_{d r}^{*}$ asymptotically.

\section{B. Step2:Speed control}

The objective is to steer the speed $\Omega$ to the desired reference $\Omega^{*}$, let $e_{\Omega}=\Omega-\Omega^{*}$ be the speed tracking error. The error dynamic of the speed is:

$$
\dot{e}_{\Omega}=\frac{P L_{m}}{L_{r} J} i_{q s} \phi_{d r}-\frac{f}{J} \Omega-\frac{T}{J}-\dot{\Omega}^{*}
$$

A Lyapunov function is defined as:

$$
V_{\Omega}=\frac{1}{2} e_{\Omega}^{2}
$$

By deriving (9) we obtain:

$$
\dot{V}_{\Omega}=e_{\Omega} \dot{e}_{\Omega}=e_{\Omega}\left(\frac{P L_{m}}{L_{r} J} i_{q s} \phi_{d r}-\frac{f}{J} \Omega-\frac{T}{J}-\dot{\Omega}^{*}\right)
$$

$i_{q s}$ is chosen as virtual element of control for stabilizing the speed, its desired value $i_{q s}^{*}$ is defined as:

$i_{q s}^{*}=\frac{J L_{r}}{L_{m} P \phi_{d r}}\left(-k_{\Omega} e_{\Omega}-k_{1} \tanh \frac{k_{1} h}{\varepsilon_{1}} e_{\Omega}+\frac{f}{J} \Omega+\dot{\Omega}^{*}\right), \phi_{d r} \neq 0$

where: $h=0.2785 . \varepsilon_{1}>0, k_{1}>0$ and $k_{\Omega}>0$ are design parameters.

By setting $i_{q s}=i_{q s}^{*}$ in (10) we get:

$$
\dot{V}_{\Omega}=e_{\Omega}\left(-k_{\Omega} e_{\Omega}-k_{1} \tanh \frac{k_{1} h}{\varepsilon_{1}} e_{\Omega}-\frac{T}{J}\right)
$$

For $k_{1}>\left|\frac{T}{J}\right|_{\max }$, we obtain:

$$
\dot{V}_{\Omega} \leq-k_{\Omega} e_{\Omega}^{2}-k_{1} \tanh \left(\frac{k_{1} h}{\varepsilon_{1}} e_{\Omega}\right) e_{\Omega}+k_{1}\left|e_{\Omega}\right|
$$

with:

$$
\left|e_{\Omega}\right|=e_{\Omega} \operatorname{signe}_{\Omega}
$$

The derivative of the Lyapunov equation (13) becomes:

$$
\dot{V}_{\Omega} \leq-k_{\Omega} e_{\Omega}^{2}-k_{1} \tanh \left(\frac{k_{1} h}{\varepsilon_{1}} e_{\Omega}\right) e_{\Omega}+k_{1} e_{\Omega} \operatorname{signe}_{\Omega}
$$

We have (see [14]):

$$
0 \leq k_{1} e_{\Omega} \text { signe }_{\Omega}-k_{1} \tanh \left(\frac{k_{1} h}{\varepsilon_{1}} e_{\Omega}\right) e_{\Omega} \leq \varepsilon_{1}
$$

The derivative of the Lyapunov function (15) becomes:

$$
\dot{V}_{\Omega} \leq-k_{\Omega} e_{\Omega}^{2}+\varepsilon_{1}
$$

This implies that the variable $e_{\Omega}$ converges to a ball whose size depends on the parameter $\varepsilon_{1}$.

\section{Step3: Currents control}

The objective is to steer the currents $i_{d s}$ and $i_{q s}$ to their desired references $i_{d s}^{*}$ and $i_{q s}^{*}$, respectively. Let $e_{d}=i_{d s}-i_{d s}^{*}$ and $e_{q}=i_{q s}-i_{q s}^{*}$ be the tracking errors of the currents, then the dynamic of the tracking errors are given by:

$$
\begin{aligned}
& \dot{e}_{d}=-a i_{d s}+\omega_{s} i_{q s}+\frac{L_{m}}{\sigma L_{s} L_{r} \tau_{r}} \phi_{d r}+\frac{V_{d s}}{\sigma L_{s}}-\frac{d i_{d s}^{*}}{d t} \\
& \dot{e}_{q}=-a i_{q s}-\omega_{s} i_{d s}-\frac{L_{m}}{\sigma L_{s} L_{r}} P \Omega \phi_{d r}+\frac{V_{q s}}{\sigma L_{s}}-\frac{d i_{q s}^{*}}{d t} \\
& \dot{e_{\phi}}=\frac{L_{m}}{\tau_{r}} e_{d}+\frac{L_{m}}{\tau_{r}} i_{d s}^{*}-\frac{1}{\tau_{r}} \phi_{d r}-\dot{\phi}_{d r}^{*} \\
& \dot{e}_{\Omega}=\frac{P L_{m}}{L_{r} J} e_{q} \phi_{d r}+\frac{P L_{m}}{L_{r} J} i_{q s}^{*} \phi_{d r}-\frac{f}{J} \Omega-\frac{T}{J}-\dot{\Omega}^{*}
\end{aligned}
$$


With:

$$
\begin{aligned}
i_{d s}^{*}= & -\frac{\tau_{r}}{L_{m}} k_{\phi} e_{\phi}+\frac{\phi_{d r}}{L_{m}}+\frac{\tau_{r}}{L_{m}} \dot{\phi}_{d r}^{*} \\
i_{q s}^{*}= & \frac{J L_{r}}{L_{m} P \phi_{d r}}\left(-k_{\Omega} e_{\Omega}-k_{1} \tanh \frac{k_{1} h}{\varepsilon_{1}} e_{\Omega}+\frac{f}{J} \Omega+\dot{\Omega}^{*}\right) \\
\frac{d i_{d s}^{*}}{d t}= & \left(\frac{1-\tau_{r} k_{\phi}}{L_{m}}\right)\left(\frac{L_{m}}{\tau_{r}} i_{d s}-\frac{\phi_{d r}}{\tau_{r}}\right)+\frac{\tau_{r} k_{\phi}}{L_{m}} \dot{\phi}_{d r}^{*}+\frac{\tau_{r}}{L_{m}} \ddot{\phi}_{d r}^{*} \\
\frac{d i_{q s}^{*}}{d t}= & \frac{J L_{r}}{L_{m} P \phi_{d r}} F_{1}\left(e_{\Omega}\right)\left(\frac{P L_{m}}{L_{r} J} i_{q s} \phi_{d r}-\frac{f}{J} \Omega\right) \\
& +F_{2}\left(e_{\Omega}, \Omega, \phi_{d r}\right)+\frac{J L_{r}}{L_{m} P \phi_{d r}}\left(\frac{f}{J}-F_{1}\left(e_{\Omega}\right)\right) \dot{\Omega}^{*} \\
& +\frac{J L_{r}}{L_{m} P \phi_{d r}} \ddot{\Omega}^{*}-\frac{L_{r} F_{1}\left(e_{\Omega}\right)}{P L_{m} \phi_{d r}} T
\end{aligned}
$$

Where:

$$
\begin{aligned}
& F_{1}\left(e_{\Omega}\right)=-k_{\Omega}-\frac{k_{1}^{2} h}{\varepsilon_{1}}\left(1-\tanh \left(\frac{k_{1} h}{\varepsilon_{1}} e_{\Omega}\right)^{2}\right)+\frac{f}{J} \\
& F_{2}\left(e_{\Omega}, \Omega, \phi_{d r}\right)=\frac{J L_{r}}{P L_{m} \phi_{d r}^{2}} \dot{\phi_{d r}} \\
& \left(k_{\Omega} e_{\Omega}+k_{1} \tanh \left(\frac{k_{1} h}{\varepsilon_{1}} e_{\Omega}\right)-\frac{f}{J} \Omega-\dot{\Omega}^{*}\right)
\end{aligned}
$$

By substituting $i_{d s}^{*}, i_{q s}^{*}, \frac{d i_{d s}^{*}}{d t}$ and $\frac{d i_{q s}^{*}}{d t}$ by their expressions, the system of the tracking errors (18) becomes:

$$
\begin{aligned}
\dot{e}_{d}= & -a i_{d s}+\omega_{s} i_{q s}+\frac{L_{m}}{\sigma L_{s} L_{r} \tau_{r}} \phi_{d r}+\frac{V_{d s}}{\sigma L_{s}} \\
& -\left(\frac{1-\tau_{r} k_{\phi}}{L_{m}}\right)\left(\frac{L_{m}}{\tau_{r}} i_{d s}-\frac{\phi_{d r}}{\tau_{r}}\right)-\frac{\tau_{r} k_{\phi}}{L_{m}} \dot{\phi}_{d r}^{*} \\
& -\frac{\tau_{r}}{L_{m}} \ddot{\phi}_{d r}^{*} \\
\dot{e}_{q}= & -a i_{q s}-\omega_{s} i_{d s}-\frac{L_{m}}{\sigma L_{s} L_{r}} P \Omega \phi_{d r}+\frac{V_{q s}}{\sigma L_{s}} \\
& -\frac{J L_{r}}{L_{m} P \phi_{d r}} F_{1}\left(e_{\Omega}\right)\left(\frac{P L_{m}}{L_{r} J} i_{q s} \phi_{d r}-\frac{f}{J} \Omega\right) \\
& -\frac{J L_{r}}{L_{m} P \phi_{d r}}\left(\frac{f}{J}-F_{1}\left(e_{\Omega}\right)\right) \dot{\Omega}^{*}-\frac{J L_{r}}{L_{m} P \phi_{d r}} \ddot{\Omega}^{*} \\
& +\frac{L_{r} F_{1}\left(e_{\Omega}\right)}{P L_{m} \phi_{d r}} T-F_{2}\left(e_{\Omega}, \Omega, \phi_{d r}\right) \\
\dot{e}_{\phi}= & -k_{\phi} e_{\phi}+\frac{L_{m}}{\tau_{r}} e_{d} \\
\dot{e}_{\Omega}= & \frac{P L_{m}}{L_{r} J} e_{q} \phi_{d r}-k_{\Omega} e_{\Omega}-k_{1} \tanh \left(\frac{k_{1} h}{\varepsilon_{1}} e_{\Omega}\right)-\frac{T}{J}
\end{aligned}
$$

Proposition 1: Let:

$$
\begin{aligned}
V_{d s}= & \sigma L_{s}\left(-k_{d} e_{d}+a i_{d s}-\frac{L_{m}}{\tau_{r}} e_{\phi}-\omega_{s} i_{q s}-\frac{L_{m}}{\sigma L_{s} L_{r} \tau_{r}} \phi_{d r}\right. \\
& \left.+\left(\frac{1-\tau_{r} k_{\phi}}{L_{m}}\right)\left(\frac{L_{m}}{\tau_{r}} i_{d s}-\frac{\phi_{d r}}{\tau_{r}}\right)+\frac{\tau_{r} k_{\phi}}{L_{m}} \dot{\phi}_{d r}^{*}+\frac{\tau_{r}}{L_{m}} \ddot{\phi}_{d r}^{*}\right) \\
V_{q s}= & \sigma L_{s}\left(-k_{q} e_{q}-k_{2} \tanh \left(\frac{k_{2} h}{\varepsilon_{2}} e_{q}\right)+a i_{q s}+\omega_{s} i_{d s}\right. \\
& +\frac{L_{m}}{\sigma L_{s} L_{r}} P \Omega \phi_{d r}-\frac{P L_{m}}{J L_{r}} e_{\Omega} \phi_{d r}+\frac{J L_{r}}{L_{m} P \phi_{d r}} F_{1}\left(e_{\Omega}\right) \\
& \left(\frac{P L_{m}}{L_{r} J} i_{q s} \phi_{d r}-\frac{f}{J} \Omega\right)+F_{2}\left(e_{\Omega}, \Omega, \phi_{d r}\right) \\
& \left.+\frac{J L_{r}}{L_{m} P \phi_{d r}}\left(\frac{f}{J}-F_{1}\left(e_{\Omega}\right)\right) \dot{\Omega}^{*}+\frac{J L_{r}}{L_{m} P \phi_{d r}} \ddot{\Omega}^{*}\right)
\end{aligned}
$$

be the actual control inputs, where $: k_{d}>0, k_{q}>0$ ,$k_{2}>0$ and $\varepsilon_{2}>0$ are design parameters. Then, if $k_{2}>\left|\frac{L_{r} F_{1}\left(e_{\Omega}\right)}{P L_{m} \phi_{d r}} T\right|_{\text {, }}$, the error variables $e_{\phi}, e_{\Omega}, e_{d}$ and $e_{q}$ are globally uniformly bounded.

Proof. By substituting the control laws (21) and (22) in (20) we get:

$$
\begin{aligned}
& \dot{e_{d}}=-k_{d} e_{d}-\frac{L_{m}}{\tau_{r}} e_{\phi} \\
& \dot{e_{q}}=-k_{q} e_{q}-k_{2} \tanh \left(\frac{k_{2} h}{\varepsilon_{2}} e_{q}\right)-\frac{P L_{m}}{J L_{r}} e_{\Omega} \phi_{d r}+\frac{L_{r} F_{1}\left(e_{\Omega}\right)}{P L_{m} \phi_{d r}} T \\
& \dot{e_{\phi}}=-k_{\phi} e_{\phi}+\frac{L_{m}}{\tau_{r}} e_{d} \\
& \dot{e_{\Omega}}= \frac{P L_{m}}{L_{r} J} e_{q} \phi_{d r}-k_{\Omega} e_{\Omega}-k_{1} \tanh \left(\frac{k_{1} h}{\varepsilon_{1}} e_{\Omega}\right)-\frac{T}{J} \\
& \text { Consider the following Lyapunov function: } \quad V=\frac{1}{2}\left(e_{\phi}^{2}+e_{\Omega}^{2}+e_{d}^{2}+e_{q}^{2}\right) \\
& \text { The derivative of } V \text { with respect to time is: } \\
& \dot{V}=e_{\phi}\left(-k_{\phi} e_{\phi}+\frac{L_{m}}{\tau_{r}} e_{d}\right) \\
& \quad+e_{\Omega}\left(\frac{P L_{m}}{L_{r} J} e_{q} \phi_{d r}-k_{\Omega} e_{\Omega}-k_{1} \tanh \left(\frac{k_{1} h}{\varepsilon_{1}} e_{\Omega}\right)-\frac{T}{J}\right) \\
& \quad+e_{d}\left(-k_{d} e_{d}-\frac{L_{m}}{\tau_{r}} e_{\phi}\right) \\
& \quad e_{q}\left(-k_{q} e_{q}-k_{2} \tanh \left(\frac{k_{2} h}{\varepsilon_{2}} e_{q}\right)-\frac{P L_{m}}{J L_{r}} e_{\Omega} \phi_{d r}+\frac{L_{r} F_{1}\left(e_{\Omega}\right)}{P L_{m} \phi_{d r}} T\right)
\end{aligned}
$$


From the step 2 we have $k_{1}>\left|\frac{T}{J}\right|_{\max }$, then the derivative of the Lyapunov function (25) becomes:

$$
\begin{aligned}
\dot{V} \leq & -k_{\phi} e_{\phi}^{2}-k_{\Omega} e_{\Omega}^{2}+\varepsilon_{1}-k_{d} e_{d}^{2}-k_{q} e_{q}^{2} \\
& -k_{2} \tanh \left(\frac{k_{2} h}{\varepsilon_{2}} e_{q}\right) e_{q}+\frac{L_{r} F_{1}\left(e_{\Omega}\right)}{P L_{m} \phi_{d r}} T e_{q}
\end{aligned}
$$

For $k_{2}>\left|\frac{L_{r} F_{1}\left(e_{\Omega}\right)}{P L_{m} \phi_{d r}} T\right|_{\text {max }}$, the derivative of the Lyapunov function (26) becomes:

$$
\dot{V} \leq-k_{\phi} e_{\phi}^{2}-k_{\Omega} e_{\Omega}^{2}-k_{d} e_{d}^{2}-k_{q} e_{q}^{2}+\varepsilon_{1}+\varepsilon_{2}
$$

This implies that the error variables $e_{\phi}, e_{\Omega}, e_{d}$ and $e_{q}$ are globally uniformly bounded.

\section{IM MODEL IN PRESENCE OF FAULT}

The presence of faults caused by rotor asymmetries (broken bars or dynamic eccentricity) and stator asymmetries (static eccentricity) induces sinusoidal components in the stator currents ( for more detail see $([4],[5])$ ), i.e:

$$
\begin{aligned}
i_{d s}= & i_{d s}+A \sin \left(\omega_{1} t+\varphi\right)+\sum_{i=1}^{N}\left[A_{i} \sin \left(\omega_{2, i} t+\varphi_{i}\right)\right. \\
& \left.+A_{-i} \sin \left(\omega_{2,-i} t+\varphi_{-i}\right)\right] \\
i_{q s}= & i_{q s}+A \cos \left(\omega_{1} t+\varphi\right)+\sum_{i=1}^{N}\left[A_{i} \cos \left(\omega_{2, i} t+\varphi_{i}\right)\right. \\
& \left.+A_{-i} \cos \left(\omega_{2,-i} t+\varphi_{-i}\right)\right]
\end{aligned}
$$

Where $i_{d s}$ and $i_{q s}$ denote the stator currents in the $(d-q)$ reference frame. The pulsations of the $2 N+1$ harmonic components depend on the kind of fault $\left(\omega_{1}\right.$ is due to the stator asymmetries, while $\omega_{2, \pm i}, i=1, \ldots, N$ are due to the rotor asymmetries). The amplitudes $A, A_{ \pm i}$ and the phases $\varphi, \varphi_{ \pm i}$ are unknown, they depend on the entity of the stator or rotor asymmetries.

The sinusoidal components generated by the presence of the rotor and stator faults can be modeled by the following exosystem $([4],[5])$ :

$$
\dot{w}=S(\varpi) w, w \in \Re^{4 N+2}
$$

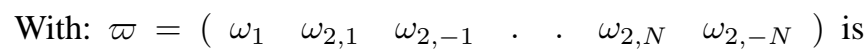
the vector of the pulsations.

$$
\begin{gathered}
S(\varpi)=\left(\begin{array}{cc}
S_{s} & 0 \\
0 & S_{r}
\end{array}\right) \\
S_{s}=\left(\begin{array}{cc}
0 & \omega_{1} \\
-\omega_{1} & 0
\end{array}\right), S_{r}=\operatorname{diag}\left(S_{r, 1}, \quad \cdot \quad \cdot \quad \cdot S_{r, N}\right) \\
S_{r, i}=\operatorname{diag}\left(\left(\begin{array}{cc}
0 & \omega_{2, i} \\
-\omega_{2, i} & 0
\end{array}\right),\left(\begin{array}{cc}
0 & \omega_{2,-i} \\
-\omega_{2,-i} & 0
\end{array}\right)\right)
\end{gathered}
$$

Where $\omega_{1}$ is the pulsation of the harmonic generated by the stator faults and $\omega_{2, \pm i}, i=1, \ldots, N$ are the pulsations of the harmonics generated by the rotor faults. The amplitudes and the phases of the harmonics are unknown, they depend on the initial state $w(0)$ of the exosystem. Then, the additive sinusoidal terms in (28) can be as a suitable combination of the exosystem state, i.e:

$$
\begin{aligned}
& i_{d s}=i_{d s}+Q_{d} w \\
& i_{q s}=i_{q s}+Q_{q} w
\end{aligned}
$$

With:

$$
\begin{aligned}
& Q_{d}=\left(\begin{array}{lllllllll}
1 & 0 & 1 & 0 & . & . & . & 1 & 0
\end{array}\right) \\
& Q_{q}=\left(\begin{array}{lllllllll}
0 & 1 & 0 & 1 & . & . & 0 & 1
\end{array}\right)
\end{aligned}
$$

By deriving (30) we get:

$$
\begin{aligned}
\frac{d i_{d s}}{d t}= & -a i_{d s}+\omega_{s} i_{q s}+\frac{L_{m}}{\sigma L_{s} L_{r} \tau_{r}} \phi_{d r}+\frac{V_{d s}}{\sigma L_{s}} \\
& +a Q_{d} w+Q_{d} S w-\omega_{s} Q_{q} w \\
\frac{d i_{q s}}{d t}= & -a i_{q s}-\omega_{s} i_{d s}-\frac{L_{m}}{\sigma L_{s} L_{r}} P \Omega \phi_{d r}+\frac{V_{q s}}{\sigma L_{s}} \\
& +a Q_{q} w+Q_{d} S w+\omega_{s} Q_{d} w
\end{aligned}
$$

Then, the IM model in presence of faults becomes:

$$
\begin{aligned}
\frac{d i_{d s}}{d t} & =-a i_{d s}+\omega_{s} i_{q s}+\frac{L_{m}}{\sigma L_{s} L_{r} \tau_{r}} \phi_{d r}+\frac{V_{d s}}{\sigma L_{s}}+\Gamma_{d}(w) \\
\frac{d i_{q s}}{d t} & =-a i_{q s}-\omega_{s} i_{d s}-\frac{L_{m}}{\sigma L_{s} L_{r}} P \Omega \phi_{d r}+\frac{V_{q s}}{\sigma L_{s}}+\Gamma_{q}(w) \\
\frac{d \phi_{d r}}{d t} & =\frac{L_{m}}{\tau_{r}} i_{d s}-\frac{1}{\tau_{r}} \phi_{d r} \\
\frac{d \Omega}{d t} & =\frac{P L_{m}}{L_{r} J} i_{q s} \phi_{d r}-\frac{f}{J} \Omega-\frac{T}{J}
\end{aligned}
$$

With:

$$
\begin{aligned}
& \Gamma_{d}(w)=a Q_{d} w+Q_{d} S w-\omega_{s} Q_{q} w \\
& \Gamma_{q}(w)=a Q_{q} w+Q_{q} S w+\omega_{s} Q_{d} w
\end{aligned}
$$

In this work the pulsations $\omega_{1}, \omega_{2, \pm i}, i=1, \ldots, N$ are assumed to be unknown. In order to reconstruct the fault effects $\Gamma_{d}(w)$ and $\Gamma_{q}(w)$ a sliding mode observer is used.

\section{FAULT RECONSTRUCTION}

Consider the system (32), where the currents $i_{d s}, i_{q s}$ and speed $\Omega$ are assumed to be measured. In order to estimate 
the fault effects $\Gamma_{d}(w), \Gamma_{q}(w)$ and the flux, a sliding mode observer is defined as:

$$
\begin{aligned}
& \frac{d \hat{i}_{d s}}{d t}=-a \hat{i}_{d s}+\hat{\omega}_{s} i_{q s}+\frac{L_{m}}{\sigma L_{s} L_{r} \tau_{r}} \hat{\phi}_{d r}+\frac{V_{d s}}{\sigma L_{s}}-u_{d} \text { signs }_{d} \\
& \frac{d \hat{i}_{q s}}{d t}=-a \hat{i}_{q s}-\hat{\omega}_{s} i_{d s}-\frac{L_{m}}{\sigma L_{s} L_{r}} P \Omega \hat{\phi}_{d r}+\frac{V_{q s}}{\sigma L_{s}}-u_{q} \text { signs }_{q} \\
& \frac{d \hat{\phi}_{d r}}{d t}=\frac{L_{m}}{\tau_{r}} i_{d s}-\frac{1}{\tau_{r}} \hat{\phi}_{d r} \\
& \hat{\omega}_{s}=P \Omega+\frac{L_{m}}{\tau_{r} \hat{\phi}_{d r}} i_{q s}
\end{aligned}
$$

Where: $\hat{i}_{d s}, \hat{i}_{q s}$ are the observed stator currents and $\hat{\phi}_{d r}$ is the flux estimate, $u_{d}>0$ and $u_{q}>0$ are design parameters. $s_{d}$ and $s_{q}$ are the sliding surfaces defined as:

$$
\begin{aligned}
& s_{d}=\hat{i}_{d s}-i_{d s} \\
& s_{q}=\hat{i}_{q s}-i_{q s}
\end{aligned}
$$

The currents and flux estimation errors are defined as: $\varepsilon_{d}=$ $s_{d}=\hat{i}_{d s}-i_{d s}, \varepsilon_{q}=s_{q}=\hat{i}_{q s}-i_{q s}$ and $\varepsilon_{\phi}=\hat{\phi}_{d r}-\phi_{d r}$, then the errors dynamics are given by:

$$
\begin{aligned}
\frac{d \varepsilon_{d}}{d t}= & -a \varepsilon_{d}+\left(\hat{\omega}_{s}-\omega_{s}\right) i_{q s}+\frac{L_{m}}{\sigma L_{s} L_{r} \tau_{r}} \varepsilon_{\phi}-\Gamma_{d}(w) \\
& -u_{d} \operatorname{sign}_{d} \\
\frac{d \varepsilon_{q}}{d t}= & -a \varepsilon_{q}-\left(\hat{\omega}_{s}-\omega_{s}\right) i_{d s}-\frac{L_{m}}{\sigma L_{s} L_{r}} P \Omega \varepsilon_{\phi}-\Gamma_{q}(w) \\
& -u_{q} \operatorname{sign} \varepsilon_{q} \\
\frac{d \varepsilon_{\phi}}{d t}= & -\frac{1}{\tau_{r}} \varepsilon_{\phi}
\end{aligned}
$$

Proposition 2: For:

$$
\begin{gathered}
u_{d}>\left|-a \varepsilon_{d}+\left(\hat{\omega}_{s}-\omega_{s}\right) i_{q s}+\frac{L_{m}}{\sigma L_{s} L_{r} \tau_{r}} \varepsilon_{\phi}-\Gamma_{d}(w)\right|_{m} \\
u_{q}>\left|-a \varepsilon_{q}-\left(\hat{\omega}_{s}-\omega_{s}\right) i_{d s}-\frac{L_{m}}{\sigma L_{s} L_{r}} P \Omega \varepsilon_{\phi}-\Gamma_{q}(w)\right|
\end{gathered}
$$

the sliding mode will occur, i.e: $\varepsilon_{d}=\dot{\varepsilon}_{d}=0$ and $\varepsilon_{q}=\dot{\varepsilon}_{q}=$ 0 , then the faults can be estimated.

Proof. Consider the following Lyapunov function:

$$
V=\frac{1}{2} \varepsilon_{d}^{2}+\frac{1}{2} \varepsilon_{q}^{2}
$$

The derivative of (38) with respect to time is:

$$
\begin{aligned}
\dot{V}= & \varepsilon_{d}\left(-a \varepsilon_{d}+\left(\hat{\omega}_{s}-\omega_{s}\right) i_{q s}+\frac{L_{m}}{\sigma L_{s} L_{r} \tau_{r}} \varepsilon_{\phi}-\Gamma_{d}(w)\right. \\
& \left.-u_{d} \operatorname{sign} \varepsilon_{d}\right)+\varepsilon_{q}\left(-a \varepsilon_{q}-\left(\hat{\omega}_{s}-\omega_{s}\right) i_{d s}\right. \\
& \left.-\frac{L_{m}}{\sigma L_{s} L_{r}} P \Omega \varepsilon_{\phi}-\Gamma_{q}(w)-u_{q} \operatorname{sign} \varepsilon_{q}\right)
\end{aligned}
$$

By choosing:

$$
\begin{gathered}
u_{d}>\left|-a \varepsilon_{d}+\left(\hat{\omega}_{s}-\omega_{s}\right) i_{q s}+\frac{L_{m}}{\sigma L_{s} L_{r} \tau_{r}} \varepsilon_{\phi}-\Gamma_{d}(w)\right|_{\text {max }} \\
u_{q}>\left|-a \varepsilon_{q}-\left(\hat{\omega}_{s}-\omega_{s}\right) i_{d s}-\frac{L_{m}}{\sigma L_{s} L_{r}} P \Omega \varepsilon_{\phi}-\Gamma_{q}(w)\right|
\end{gathered}
$$

the sliding mode occurs, i.e: $\varepsilon_{d}=\dot{\varepsilon}_{d}=0$ and $\varepsilon_{q}=\dot{\varepsilon}_{q}=0$.

Therefore the equations (35) become:

$$
\begin{gathered}
\left(\hat{\omega}_{s}-\omega_{s}\right) i_{q s}+\frac{L_{m}}{\sigma L_{s} L_{r} \tau_{r}} \varepsilon_{\phi}-\Gamma_{d}(w)-u_{d} \operatorname{sign}_{e q} \varepsilon_{d}=0 \\
-\left(\hat{\omega}_{s}-\omega_{s}\right) i_{d s}-\frac{L_{m}}{\sigma L_{s} L_{r}} P \Omega \varepsilon_{\phi}-\Gamma_{q}(w)-u_{q} \operatorname{sign}_{e q} \varepsilon_{q}=0 \\
\frac{d \varepsilon_{\phi}}{d t}=-\frac{1}{\tau_{r}} \varepsilon_{\phi}
\end{gathered}
$$

Equation (42) shows that $\varepsilon_{\phi}$ converges to zero as $t \rightarrow \infty$, then $\hat{\omega}_{s} \rightarrow \omega_{s}$ and the estimates of the faults are given by:

$$
\begin{aligned}
& \hat{\Gamma}_{d}=-u_{d} \operatorname{sign}_{e q}\left(\varepsilon_{d}\right) \\
& \hat{\Gamma}_{q}=-u_{q} \operatorname{sign}_{e q}\left(\varepsilon_{q}\right)
\end{aligned}
$$

Remark 1: The function $\operatorname{sign}_{e q}$ represents the average value of the sign function, it can be obtained by the use of a low passe filter or by a continuous approximation of the sign function. Here the sign function is approximated by an hyperbolic tanh function.

\section{DESIGN OF FAULT TOLERANT CONTROL}

The structure of the global controller is given by:

$$
\begin{aligned}
& V_{d s}=V_{d s n}+V_{d f} \\
& V_{q s}=V_{q s n}+V_{q f}
\end{aligned}
$$

with $V_{d s n}$ and $V_{q s n}$ are the backstepping control laws (21) and (22) designed in un-faulty mode $(w(t)=0)$ to steer the tracking errors to zero and to compensate the load disturbance.

$V_{d f}$ and $V_{q f}$ are additional control laws (compensation units) that will be designed in order to compensate the faults.

Proposition 3: Let $V_{d s n}$ and $V_{q s n}$ be the backstepping control laws given by (21) and (22) and let:

$$
\begin{aligned}
& V_{d f}=-\sigma L_{s} \hat{\Gamma}_{d} \\
& V_{q f}=-\sigma L_{s} \hat{\Gamma}_{q}
\end{aligned}
$$

be the additional control laws, where the estimate $\hat{\Gamma}_{d}, \hat{\Gamma}_{q}$ are given by (43). Then the faults are compensated. 
Proof. The dynamics of the tracking errors are given by:

$$
\begin{aligned}
\frac{d e_{d}}{d t}= & -a i_{d s}+\omega_{s} i_{q s}+\frac{L_{m}}{\sigma L_{s} L_{r} \tau_{r}} \phi_{d r}+\frac{V_{d s}}{\sigma L_{s}} \\
& -\left(\frac{1-\tau_{r} k_{\phi}}{L_{m}}\right)\left(\frac{L_{m}}{\tau_{r}} i_{d s}-\frac{\phi_{d r}}{\tau_{r}}\right)-\frac{\tau_{r} k_{\phi}}{L_{m}} \dot{\phi}_{d r}^{*} \\
& -\frac{\tau_{r}}{L_{m}} \ddot{\phi}_{d r}^{*}+\Gamma_{d}(w) \\
\frac{d e_{q}}{d t}= & -a i_{q s}-\omega_{s} i_{d s}-\frac{L_{m}}{\sigma L_{s} L_{r}} P \Omega \phi_{d r}+\frac{V_{q s}}{\sigma L_{s}} \\
& -\frac{J L_{r}}{L_{m} P \phi_{d r}} F_{1}\left(e_{\Omega}\right)\left(\frac{P L_{m}}{L_{r} J} i_{q s} \phi_{d r}-\frac{f}{J} \Omega\right) \\
& -\frac{J L_{r}}{L_{m} P \phi_{d r}}\left(\frac{f}{J}-F_{1}\left(e_{\Omega}\right)\right) \dot{\Omega}^{*}-\frac{J L_{r}}{L_{m} P \phi_{d r}} \ddot{\Omega}^{*} \\
& +\frac{L_{r} F_{1}\left(e_{\Omega}\right)}{P L_{m} \phi_{d r}} T-F_{2}\left(e_{\Omega}, \Omega, \phi_{d r}\right)+\Gamma_{q}(w) \\
\frac{d e_{\phi}}{d t}= & -k_{\phi} e_{\phi}+\frac{L_{m}}{\tau_{r}} e_{d} \\
\frac{d e_{\Omega}}{d t}= & \frac{P L_{m}}{L_{r} J} e_{q} \phi_{d r}-k_{\Omega} e_{\Omega}-k_{1} \tanh \left(\frac{k_{1} h}{\varepsilon_{1}} e_{\Omega}\right)-\frac{T}{J}
\end{aligned}
$$

By substituting (44) in (47) we get:

$$
\begin{aligned}
\frac{d e_{d}}{d t}= & -k_{d} e_{d}-\frac{L_{m}}{\tau_{r}} e_{\phi}+\Gamma_{d}(w)-\hat{\Gamma}_{d}(w) \\
\frac{d e_{q}}{d t}= & -k_{q} e_{q}-k_{2} \tanh \left(\frac{k_{2} h}{\varepsilon_{2}} e_{q}\right)-\frac{P L_{m}}{J L_{r}} e_{\Omega} \phi_{d r} \\
& +\frac{L_{r} F_{1}\left(e_{\Omega}\right)}{P L_{m} \phi_{d r}} T+\Gamma_{q}(w)-\hat{\Gamma}_{q}(w) \\
\frac{d e_{\phi}}{d t}= & -k_{\phi} e_{\phi}+\frac{L_{m}}{\tau_{r}} e_{d} \\
\frac{d e_{\Omega}}{d t}= & \frac{P L_{m}}{L_{r} J} e_{q} \phi_{d r}-k_{\Omega} e_{\Omega}-k_{1} \tanh \left(\frac{k_{1} h}{\varepsilon_{1}} e_{\Omega}\right)-\frac{T}{J}
\end{aligned}
$$

Section (5) shows that $\hat{\Gamma}_{d}(w) \rightarrow \Gamma_{d}(w)$ and $\hat{\Gamma}_{q}(w) \rightarrow \Gamma_{q}(w)$ then the faults are compensated and the resulting closed loop system is stable. Its stability is proved in Section 3 by the Lyapunov function (24).

\section{SIMULATION RESULTS}

Numerical simulations have been performed to validate the proposed control scheme. The induction motor parameters are given in the appendix. The controller parameters are chosen as follows: $k_{d}=650, k_{q}=500, k_{\Omega}=0.5, k_{\phi}=$ $25, k_{1}=300, k_{2}=300, u_{d}=26000, u_{q}=26000$. The speed and flux references are fixed at $\Omega_{*}=100 \mathrm{rad} / \mathrm{s}$ and $\phi_{d r}^{*}=0.9 \mathrm{~Wb}$, respectively, also a load disturbance $T=3 N . m$ is applied. Figure 1 shows the responses of the induction motor in the absence of faults in case just the backstepping controller is present in the loop. The speed and the flux trajectories converge to their desired references and the load disturbance is rejected. Figure 2 shows the responses of the induction motor controlled by the backstepping controller when stator and rotor faults occur. The IM responses exhibit oscillations and deviation from their desired references. Figure 3 shows the responses of the IM in case the backstepping controller is augmented with the fault compensation units. The effectiveness of the compensation units in compensating the effect of the faults is evident. Figure 4 shows a good estimation of the faults. In order to test the robustness of the proposed control scheme, the variations of the rotor resistance $R_{r}$ and stator resistance $R_{s}$ are introduced. the simulation results are given by the figure 5. We see that the proposed controller is insensitive to the rotor and stator resistance variations.

\section{CONCLUSION}

In this paper a sliding mode observer based approach to fault compensation for induction motors has been presented. In absence of faults a backstepping controller permits to steer the flux and the speed variables to their desired references and to reject the load disturbance, however the presence of faults degradates the performances of the induction motor. In order, to detect and estimate the faults a sliding mode observer is used. The use of a sliding mode observer provides a good estimation of the faults. Then, additional control laws based on the resulting faults estimates permit to eliminate the effect of the faults. 


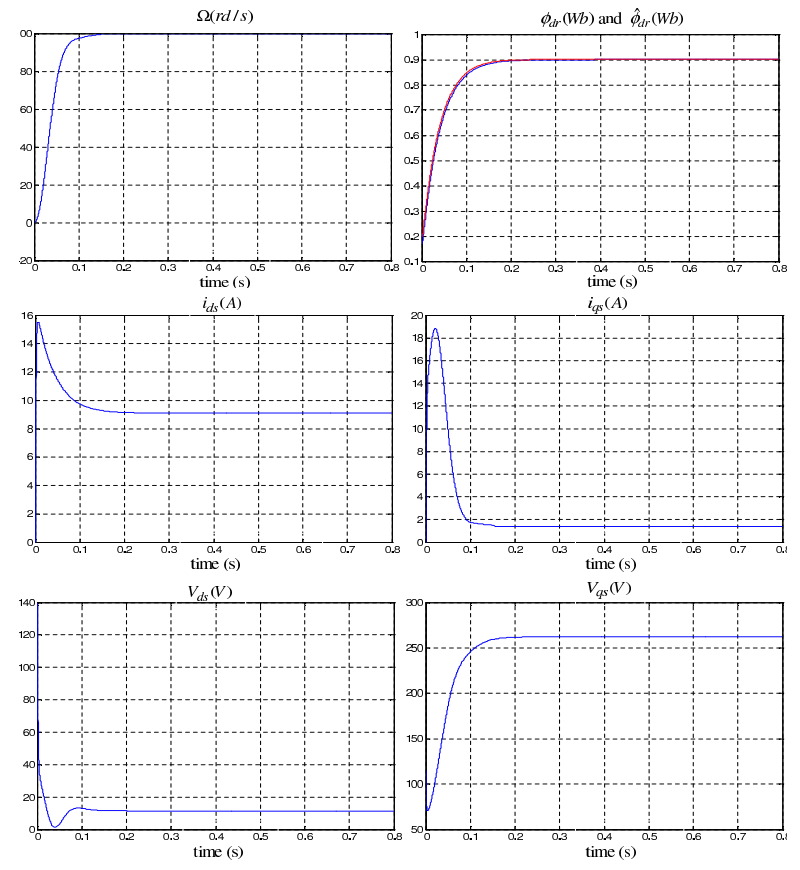

Fig. 1. Responses of the IM controlled by the backstepping controller in un-faulty mode

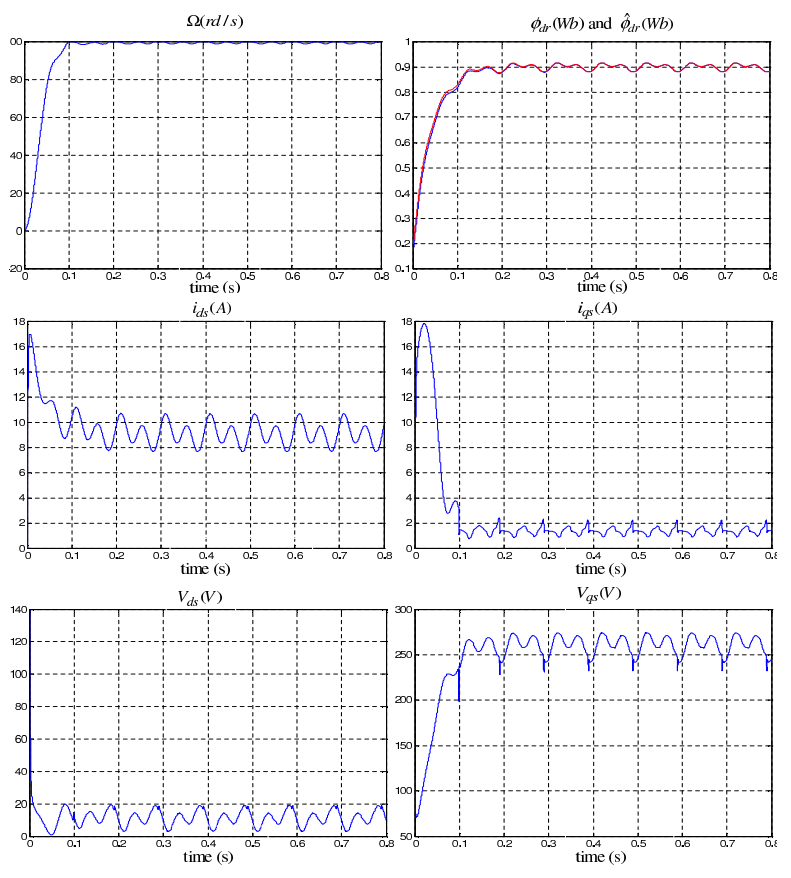

Fig. 2. Responses of the IM controlled by the backstepping controller when faults occur
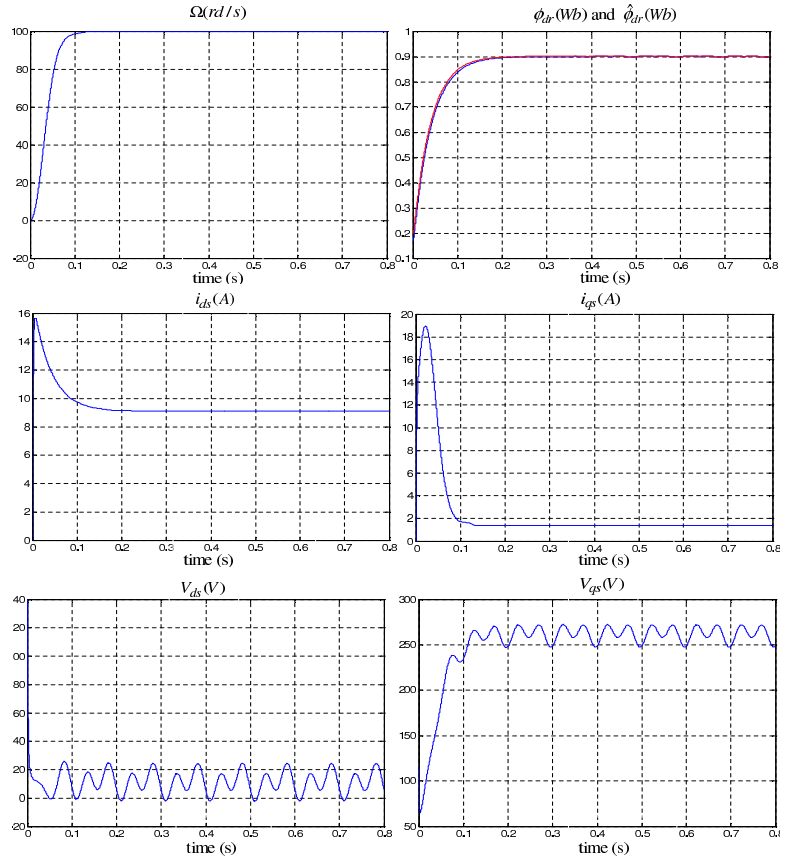

Fig. 3. Responses of the IM in case the backstepping controller is augmented with the fault compensation units
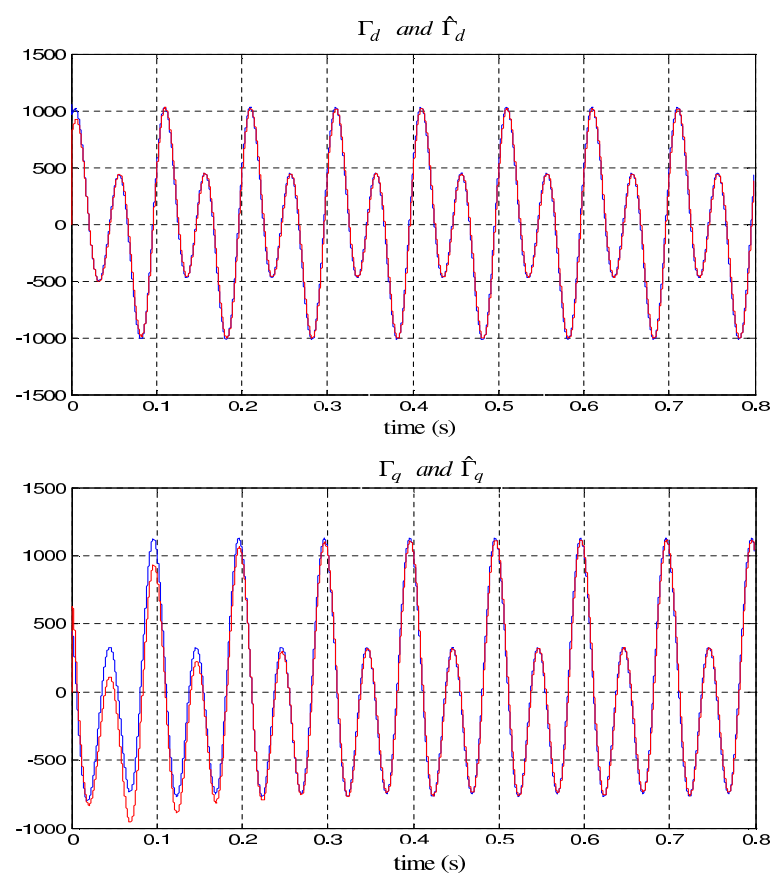

Fig. 4. The faults and their estimates 


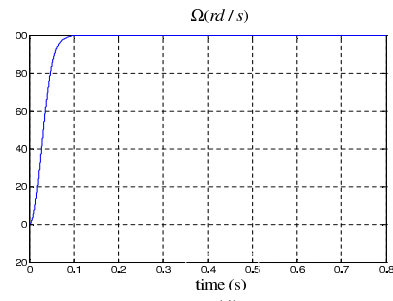

$i_{d s}(A)$
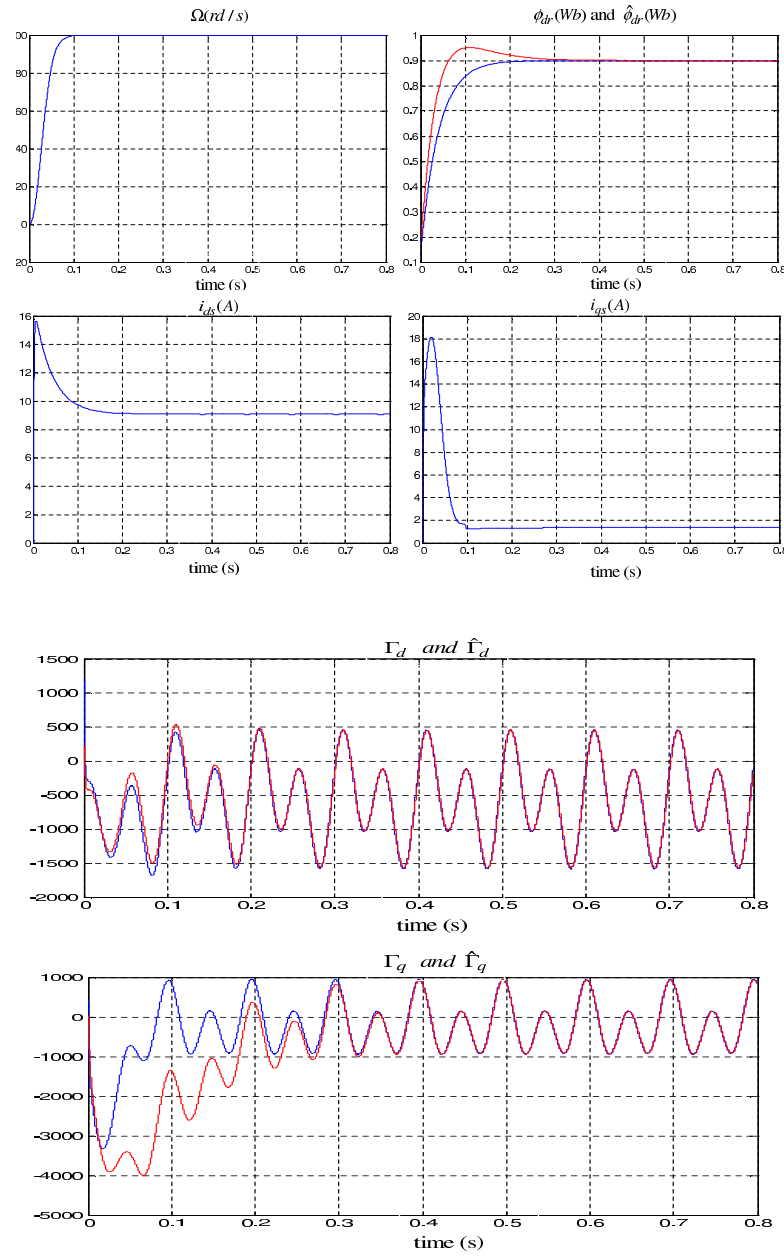

Fig. 5. Responses of the IM with an increase of $+50 \% R_{r}$ and $+50 \% R_{s}$

\section{ApPEndiX}

The induction motor used in this work is a $1.5 \mathrm{KW}, U=220 \mathrm{~V}, 50 \mathrm{~Hz}, I_{n}=7.5 \mathrm{~A}$. The parameters are: $R_{s}=1.633 \Omega, R_{r}=0.93 \Omega, L_{r}=0.076 H, L_{s}=$ $0.142 \mathrm{H}, L_{m}=0.099 \mathrm{H}, J=0.0111 \mathrm{Kg} \cdot \mathrm{m}^{2}, f=$ $0.0018 \mathrm{~N} . \mathrm{m} / \mathrm{rad} / \mathrm{s}$ and $P=2$.

\section{REFERENCES}

[1] M. Blanke, R. Izadi-Zamanabadi, S. Bogh and C. Lunau, Fault tolerant control systems- a holistic view, Control engineering Practice, pp. 693702, 1997.

[2] M. Blanke, M. Staroswiecki and N. Wu, Concepts and methods in fault-tolerant control, Proceedings of the american control conference, Arlington, pp. 2606-2020, 2001.

[3] P. Mhaskar, A. Gani, PD. Christofides, Fault-tolerant control of nonlinear processes:Performance-based reconfiguration and robustness, Int J Robust Nonlinear Control, pp. 91-111, 2006.
[4] C. Bonivento , A. Isidori ,L. Marconi, A. Paoli , Implicit fault tolerant control: Application to induction motors. IFAC, 2002.

[5] C. Bonivento , A. Isidori ,L. Marconi and A. Paoli , Implicit fault tolerant control: Application to induction motors. Automatica, 40, pp. 355-371, 2004.

[6] F. Tahami, A. Shojaei and D. A. Khatir, A diversity based reconfigurable method for fault tolerant control of induction motors, International Symposium on Power Electronics, Electrical Drives, Automation and Motion, pp.66-71, 2006.

[7] R. L. A. Ribeiro, C. B. Jacobina, E. R. C. Silva and A.M.N.Lima, Compensation Strategies in the PWM- VSI Topology for a Fault Tolerant Induction Motor Drive System, Atlanta, 2003.

[8] J. M. Miller and C. Morrison, Fault-tolerant operation of induction motor drives with automatic controller reconfiguration, in conf.Rec.IEEE IEMDC, pp.156-162, 2001.

[9] C. Edwards, S. K. Spurgeon and R. J. Patton, Sliding mode observers for fault detection and isolation, Automatica, 36, 541-553, 2000.

[10] C. Tan and C. Edwards, Sliding mode observers for detection and reconstruction of sensor faults, Automatica, 38, 1815-1821, 2003.

[11] T. Floquet, J-P. Barbot, W. Perruquetti and M. Djemai, On the robust fault detection via a sliding mode disturbance observer, INT. J. Control, Vol.77, No.7, pp. 622-629, 2004.

[12] C. Canudas de Wit, Commande des moteurs asynchrones 2, Optimisation, discrtisation et observateurs, Edition Hermes Science, Paris, 2000.

[13] C. Chen, Backstepping control design and its applications to vehicle Lateral control in automated highway systems, Dissertation, University of California at Berkeley, 1996.

[14] M. Polycarpou and P. Ioannou, A Robust Adaptive Nonlinear Control Design", Proceedings of American Control Conference, pp. 13651369, 1993. 